[Agr. Biol. Chem., Vol. 31, No. 12, p. 1474 1481, 1967]

\title{
Studies on Changes of Protein by Dye Sensitized Photooxidation
}

\author{
Part I. Photodecomposition of Amino Acid \\ Residues of Ovalbumin* \\ By Toshiharu Gomyo and Yosito SAKuraI** \\ Department of Agricultural Chemistry, Faculty of Agriculture, \\ The University of Tokyo \\ Received July 8, 1967
}

\begin{abstract}
Ovalbumin solution was illuminated with W-lamp in the presence of methylene blue as a sensitizer. The photosusceptibility of amino acid residues of ovalbumin was found to be in the following order, Try $>$ His $>$ Met $>$ Ser. Sulfhydryl group, however, was scarcely decomposed until about eighty per cent of tryptophan residue was decomposed. Free tryptophan was much faster in photodecomposition than bound tryptophan in ovalbumin because of steric and protective effects.

With the increase of methylene blue in the lower concentration than $1.0 \times 10^{-5} \mathrm{M}$, the relative quantum yield for the decomposition of tryptophan residue increased. This was elucidated on the assumption that the adsorption of dye molecules on ovalbumin brought about the loss of photosensitizing ability. The $\mathrm{pH}$ dependence also was investigated and some other factors influencing photosensitized oxidation were examined.
\end{abstract}

\section{INTRODUCTION}

It is well known that amino acids and proteins are photodynamically attacked under aerobic condition. Particularly, tryptophan, tyrosine, histidine, methionine and cystein are susceptible to photosensitized decomposition. ${ }^{1 \sim 4}$ According to the reports previously published, peptide linkages of proteins do not undergo any scission by photosensitization of visible light $(>400 \mathrm{~m} \mu) .^{5}$ Therefore, changes of properties of proteins may be caused mainly

* This report was presented at the Annual Meeting of the Agricultural Chemical Society of Japan, April, 3, 1966.

** Present address: Nihon Women's University, Tokyo, Japan.

1) L. Weil, A. R. Buchert and J. Maher, Arch. Biochem. Biophys., 40, 245 (1952).

2) L. Weil, S. James and A. R. Buchert, ibid., 46, 266 (1952).

3) L. Weil and T.S. Seibles, ibid., 54, 368 (1955).

4) Z. Vodrazka and O. Mch, Biochim Biophys. Acta, 49, 495 (1961).

5) R. Egan, H. O. Michel, R. Schlueter and B. J. Jandorf, Arch. Biochem. Biophys, 66, 366 (1957). by decomposition of their side chains which include indole, phenol, imidazole and sulfur containing groups.

Many investigations have been carried out to elucidate the photooxidation products of free amino acids mentioned above. For example, kynulenine, 3-oxy-kynulenine and anthranilic acid have been found from tryptophan, DOPA (3, 4-dioxyphenylalanine) from tyrosine, alanine and urea from histidine, methioninesulfone from methionine and cysteic acid from cystein. ${ }^{6 \sim 111}$

The products from those amino acids are

6) J. Tabone, Bull. Soc. Chim. Biol., 31, 1270 (1949).

7) G. Matsuda, Nagasaki Igakkai Zassi, xxviii, 811 (1953).

8) Z. Yoshida and M. Kato, Nippon Kagaku Kaishi, 75, 106 (1954).

9) Y. Obata and S. Sakamura, J. Agr. Chem. Soc. Japan, 28, 768 (1954).

10) H. Hara, ibid., 34, 493 (1960).

11) R. Ale and S. C. Agarwala, Indian J. Biochem., 2, 271 (1965). 
varied depending on such experimental conditions as $\mathrm{pH}$, sensitizers used and so on." On the other hand, each of these products may be considered to be a sort of intermediate which is affected by further decomposition. Although various explanations have been proposed for the mechanisms of photodecomposition of amino acids, ${ }^{12}$ the decisive theory has not yet been accomplished.

The photosensitized degradation of enzyme proteins loses the activities with the destruction of photosusceptible amino acid residues. ${ }^{131}$ This fact is claimed as very useful for the elucidation of active sites of enzmye proteins. ${ }^{14}$ Nothing is, however, clear about amino acid residues modified through photosensitized oxidation of proteins. Complete explanation of proteins photodynamically modified will not be given until the structure of products is made clear.

The present paper deals with the extent of decomposition of amino acid residues of ovalbumin under various condition. Because tryptophan residue was so remarkable in decomposition that the photooxidation of ovalbumin was evaluated by the decrease of tryptophan residue. In the following, several conditions governing to photodecomposition of amino acid residues will be shown.

\section{EXPERIMENTALS}

Preparation of ovalbumin. Following the method of Kekwick et al., 15) the authors obtained ovalbumin recrystallized three times from sodium sulfate solution, dialized against distilled water and freezed into dry powder. This gave a single peak in the ultracentrifugal pattern and three peaks in the Tiselius electrophoretic pattern. The powder was stored in a colored bottle under a dry and cool condition.

12) G. Matsuda, Nagasaki Igakkai Zassi, xxviii, 438 (1953); G. O. Schenck, Angew. Chem., 69, 579 (1965); L. Weil, Arch. Biochem. Biophys., 110, 57 (1965).

13) D. Shugar, Biochim. Biophys. Acta, 8, 302 (1952).

14) C. A. Chiron and J.d. Spikes, U.S. Atomic Energy Commission Contract, No. AT (11-1), 875 (1963).

15) R. A. Kekwick and R. K. Cannan, Biochem. J., 30, 232 (1936).
Sensitizer. Methylene blue purchased from Kanto Chemical Co. Ltd. was used without further purification.

Illumination procedure. The light sources were candecent lamps from Tokyo Shibaura Denki Co. Ltd. The power was adjusted with a voltage stabilizer from Fuji Seimitsu Denki Co. Ltd., $100 \mathrm{~V}, 200 \mathrm{~W}$. The reaction solution in a glass tube $(18 \times 170 \mathrm{~mm})$ was put in a thermostat vessel which has a glass window and set at the constant temperature of $30^{\circ} \mathrm{C}$ by circulating cold water. The distance between the surface of the light source bulb and the center of the reaction tube was $11 \mathrm{~cm}$.

Composition of reaction solution. Unless stated otherwise, the reaction mixture was prepared as follows. To $9.0 \mathrm{ml}$ of $0.5 \%$ ovalbumin solution in $0.1 \mathrm{M}$ acetate buffer of $\mathrm{pH} 6.0$ was added $1.0 \mathrm{ml}$ of $10^{-3} \mathrm{M}$ methylene blue solution in the same buffer just before illumination.

Assays of amino acid residues of ovalbumin.

Prior to analysis, all samples were treated with a cation exchanger (Amberlite IR 120) at $\mathrm{pH} 8.0$ to remove dye. If necessary, the samples were hydrolyzed according to the method of Ceriotti et al.16) Tryptophan was measured by the method of Spies et al.,17) tyrosine by $\alpha$-nitroso- $\beta$-naphtol procedure, ${ }^{16)}$ histidine by $p$-nitrobenzoyl chloride procedure, 18 ) methionine by the method of Bakey et al.,19) sulfhydryl group by N-ethylmaleimide procedure, ${ }^{20}$ ) and arginin by the Sakaguchi's reaction.21) In addition all the constituents of ovalbumin were analyzed by using an auto-analyzer for amino acids.

Calculation. According to the method of Bellin et al.22) an approximation to the actual quantum yield was given as follows:

$\Phi$ relative

decrease of amino acid residues $(\%)$

time of illumination(min) $\times$ fraction of light absorbed

The fraction of light absorbed by the solution is

16) G. Ceriotti and L. Spandrio, Biochem. J., 66, 607 (1957).

17) J. R. Spies and D. C. Chambers, Anal. Chem., 20, 30 (1948).

18) R. W. Cowgill, ibid., 27, 1521 (1955)

19) B. Bakey and G. Toennies, J. Biol. Chem., 188, I (1951).

20) J. Leslie, D. L. Williams and G. Gorin, Anal. Biochem., 3, 257 (1962).

21 C. J. Weber, J. Biol. Chem, , 86, 217 (1930)

22) J. S. Bellin and G. Entner, Photochem. Photobiol., 5, 251 (1966). 
equal to $\left(1-e^{-\varepsilon c d}\right)$, where $\varepsilon$ is the molar extinction coefficient of the dye used as sensitizer at its absorption maximum, $c$ is the concentration of the dye in moles per liter, and $d$ is the path length of the reaction tube $(\mathrm{cm})$. The path length used in this experiment was evaluated approxmately as $\pi^{r / 2}(\mathrm{~cm})$ where $r$ was a diameter of a reaction tube.

\section{RESULTS AND DISCUSSION}

Decrease of Amino Acid Contents of Ovalbumin.

Aromatic and sulfur containing amino acid residues of ovalbumin are more remarkably decomposed than aliphatie ones except serine and aspartic residues (Table I). Phenyl alanyl

Ttable I. Change of Amino Acid Contents OF OVALBUMIN

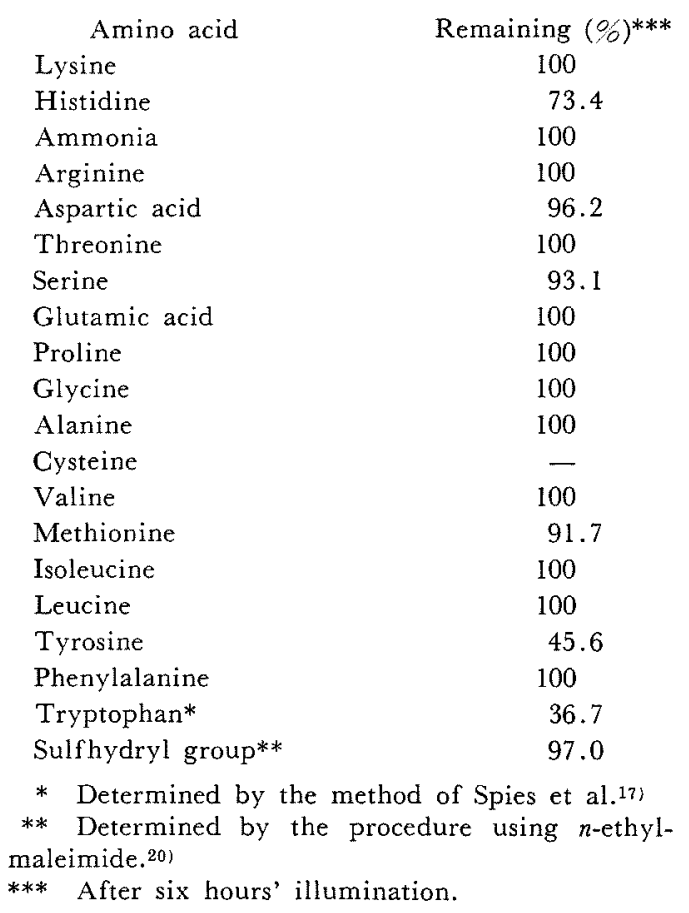

residue, however, is excluded from photosusceptible aromatic amino acids. Apart from the loss of serine and aspartic acid, these results are well corresponded to those obtained from other proteins. But the extent of the loss of amino acid residues seems to be dependent on the kind of proteins. ${ }^{10}$ The unexpected

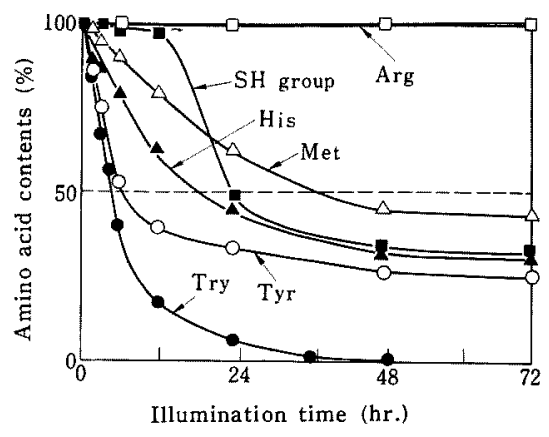

FIG. 1. Effect of Illumination Time on Decrease of Amino Acid Contents of Ovalbumin.

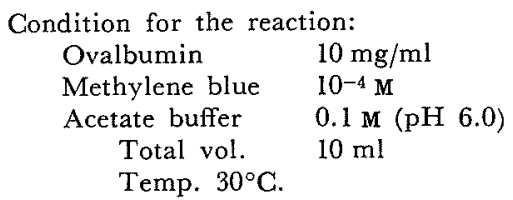

loss of serine and aspartic residues is now under question. Six-hour illumination caused the loss of $63 \%$ of tryptophan residue, 54\% of tyrosine residue, $27 \%$ of histidine residue, $8 \%$ of methionine residue and $7 \%$ of serine residue, respectively. Sulfhydryl group however, lost only $3 \%$. This fact appears to be very strange as compared with the decomposition of free cysteine which is known to be one of the most photosusceptible amino acids.

Sulfhydryl group, however, has an abrupt turn in decrease after illumination for about twelve hours as shown in Fig. 1. This group may be protected from photodynamic attack by the presence of other amino acid residues. The decomposition of sulfhydryl group seems to be facilitated by that of some other residues. The rate of the decomposition of amino acid residues may be effectively influenced by their steric positions in protein structure.

Histidine residue, which has been considered to play as important role in the action of hydrolytic enzymes, ${ }^{14}$ is is ually very rapid in the rate of decomposition. This suggests that histidine residues locates on the surface of hydrolytic enzyme to catalize effectively 
hydrolysis. At $\mathrm{pH} 6.0$, the rate of the decomposition of histidine (free amino acid) is larger than that of tryptophan (free) and tyrosine (free) when illuminated in the presence of methylene blue according to our preliminary experiment. Therefore, it is probable that histidine residues of ovalbumin are concealed in protein structure which prevents these residues from photosensitized oxidation.

Both tryptophan and tyrosine residues decrease linearly up to about six hours and the former completely disappeares at about thirtyfive hours of illumination. Tyrosine, histidine, methionine and sulfhydryl group show the resistance against decomposition after about twenty-four hours of illumination. Especially, this tendency is remarkable in the case of sulfhydryl group. It may be natural to put some classes in the same amino acid residues according to the extent of susceptibility to photosensitized decomposition. Koshland et al. classified 11 histidine residues of phosphoglucomutase into two portions, the one corresponding to 5 histidine residues which are decomposed 'rapidly' and the other to 6 residues are decomposed 'slowly' 231

Comparison of Free Tryptophan with Tryptophan Residue of Ovalbumin in Its Photodecomposition.

The free tryptophan is decomposed ten times faster than the tryptophan bound to protein at the initial period of illumination (Fig. 2). The both test solutions were prepared to be equal in respect to molar concentration of tryptophan. This difference between the free tryptophan and the bound one may be based on the protective effect of other amino acid residues than tryptophan and on the steric hindrance of protein skeleton with globular shape. This suggestion will be supported in the following two experiments.

Fig. 3 shows that tryptophan is protected from photodecomposition by the presence of

23) D. E. Koshland, W. J. Ray and M. J. Erwin, Fed. Proc., 17, 1145 (1958).

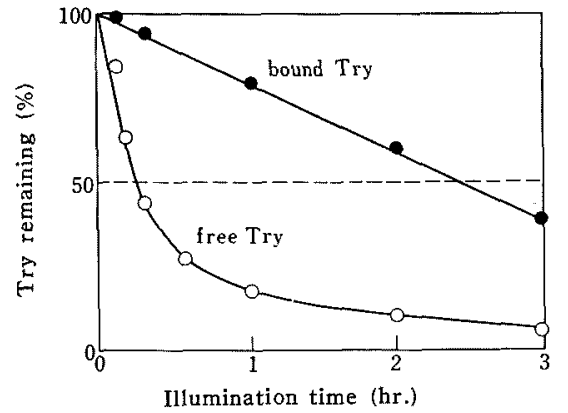

FIG. 2. Comparison of Free Tryptophan with Bound One in the Rate of Photosensitized Decomposition at $\mathrm{pH} 6.0,30^{\circ} \mathrm{C}$.

Condition for the reaction:

Try (free and bound*)

Methylene blue $\quad 10^{-4} \mathrm{M}$

Acetate buffer $\quad 0.1 \mathrm{M}(\mathrm{pH} 6.0)$

Total vol.

$10 \mathrm{ml}$

* The conc. of bound try was estimated from the content of Try residue in ovalbumin.

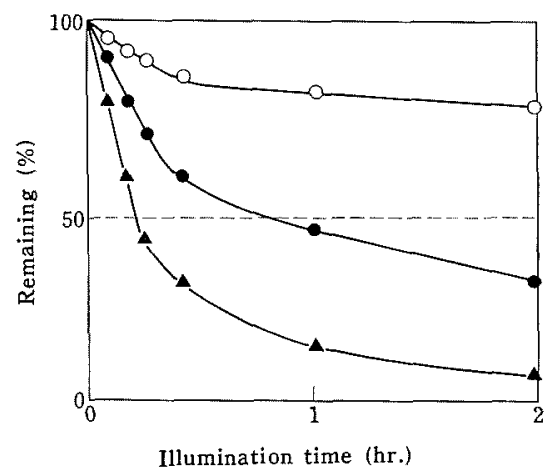

FIG. 3. Protective Effect of Tyr on the Decomposition of Try at $\mathrm{pH} 6.0,30^{\circ} \mathrm{C}$.

$$
\begin{aligned}
& \begin{array}{l}
-\boldsymbol{\Delta}-\operatorname{Try}\left(4 \times 10^{-4} \mathbf{M}\right) \\
\operatorname{Try}\left(4 \times 10^{-4} \mathbf{M}\right)+\operatorname{Tyr}\left(12 \times 10^{-3} \mathrm{M}\right)
\end{array} \\
& \begin{cases}- \text { Try remaining } \\
-0 & \text { Tyr remaining. }\end{cases}
\end{aligned}
$$

tyrosine. The amount of lost tyrosine is chemically equivalent to decreased amount of tryptophan by decomposition at the initial stage. Therefore, tryptophan and tyrosine may compete for a certain active species which has been considered to be excited dye, oxygen-dye complex, activated oxygen or some others. 


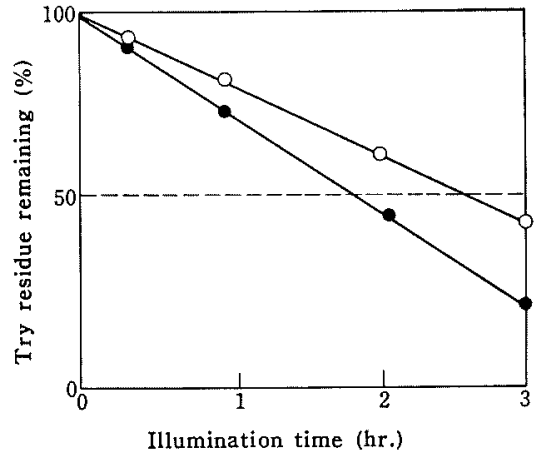

FIG. 4. Effect of Urea on the Decompesition of Try Residue of Ovalbumin at $\mathrm{pH} 6.0,30^{\circ} \mathrm{C}$.

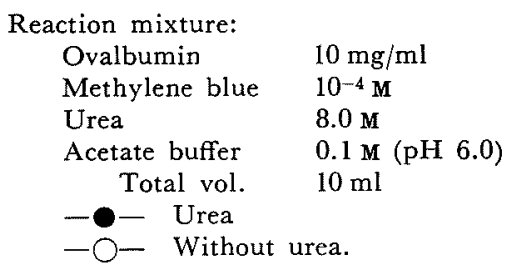

By means of conventional method, the samples were treated with $8 \mathrm{~m}$ urea which did not affect the rate of decomposition of tryptophan in the preliminary test. As shown in Fig. 4, this treatment raises the rate of the decomposition of tryptophan residue. Tryptophan residue is considered to be easily attacked through randomizing protein structure in which tryptophan residue is folded. ${ }^{241}$

From the results of these experiments the authors have come to the conclusion that the difference between the rates of both free tryptophan and bound tryptophan is due to the competitive effect and the steric effect at least.

Effect of Methylene Blue Concentration on the Rate of Decomposition of Tryptophan Residue of Ovalbumin.

With the increase of methylene blue in concentration, the decomposition of tryptophan residue gets faster up to the concentration of $0.4 \times 10^{-4} \mathrm{M}$. At this concentration of methy-

24) S. Kim, J. Agr. Chem. Soc. Japan, 40, 37 (1966).

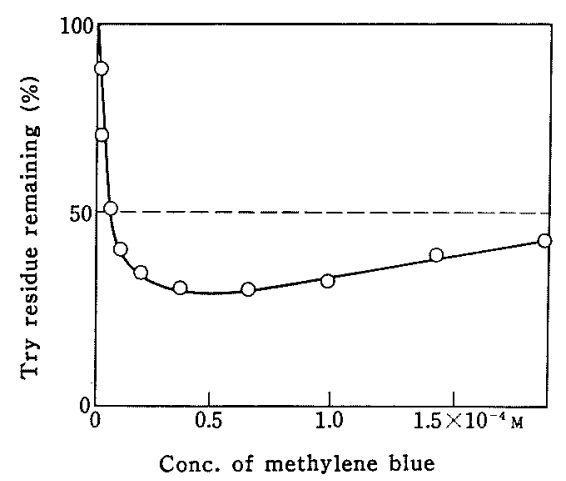

FIG. 5. Effect of Concentration of Dye on the Decomposition of Try residue of Ovalbumin at $\mathrm{pH}$ $6.0,30^{\circ} \mathrm{C}$.

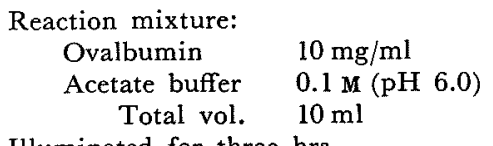

lene blue there is an abrupt turn (Fig. 5). The curve shown in this figure consists of two parts: $\mathrm{C}_{1}, \mathrm{C}_{2}$. When low in concentration of methylene blue $\left(\mathrm{C}_{1}\right)$, the rate of decomposition is proportional to the increase of the concentration of methylene blue. Here methylene blue may join the rate regulating stage of photosensitezed oxidation. The maximum rate of decomposition is at the concentration of $0.4 \times 10^{-4} \mathrm{M}$. Beyond that concentration, the rate gradually decreases. This gradual decrease may be due partly to concentration quenching because of too high concentration of dye. ${ }^{251}$ Further investigation about concentration effect on the decomposition of tryptophan residue was carried out with respect to relative quantum yield (Fig. 6). The quantum yield of the decomposition of tryptophan residue increases as the amount of sensitizer used increases up to about $1.0 \times 10^{-5} \mathrm{M}$. Above this concentration, the quantum yield remains almost constant. This result is well corresponded to the report about the quantum

25) S. Kim, J. Agr. Chem. Soc. Japan, 39, 10 (1965). 


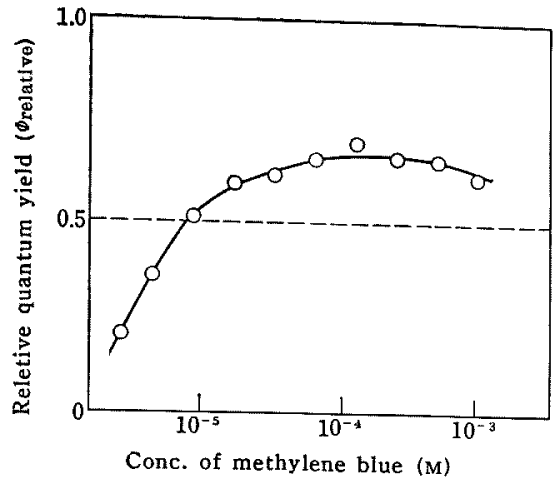

FIG. 6. Dependence of Relative Quantum Yield on Conc. of Methylene Blue.

The experimental conditions were the same as shown in Fig. 5.

yield for the denaturation of ovalbumin photosensitized by thionine similar with methylene blue in structure. ${ }^{21}$ This similarity indicates the identity of the mechanism for producing active species playing an important role in photosensitezed oxidation. Oster et al., carried out the photosensitized oxidation of $p$-toluenediamine with proflavin. ${ }^{261}$ They showed that the quantum yield decreased with increasing dye concentration according to Stern-Volmer relation. This differs from our result obtained in the photosensitized oxidation of ovalbumin. The increment of the relative quantum yield up to the concentration of $1.0 \times 10^{-5} \mathrm{M}$ may be characteristic of protein on which dye molecules can be adsorbed. In spite of concentration quenching, this increase can be explained on the assumption that dye molecules adsorbed on the surface of protein are inactive for photosensitization and the light absorption of dye in the complex is as large as that of free dys as follows. If the equilibrium equation is expressed as $K=(P) \cdot(D) /(P D)$, where $K$ is equilibrium constant, $(P)$ is protein concentration, $(D)$ is dye concentration and $(P D)$ is dye protein complex

26) G. Oster, J. S. Bellin, R. W. Kimball and M. E. Schrader, J. Am. Chem. Soc., 81, 5095 (1959). concentration,

$$
K=\frac{\left\{(P)_{0}-(D)_{0}+(D)\right\}(D)}{(D)_{0}-(D)}
$$

where $(P)_{0}$ is the initial concentration of protein, $(D)_{0}$ is the initial concentration of dye, is obtained from the following relations:

$$
\begin{gathered}
(P)=(P)_{0}-(D)_{0}+(D) \\
(P D)=(D)_{0}-(D)
\end{gathered}
$$

By putting $R \cdot(D)_{0}$ for $(D)$, where $R$ is the ratio $(D) /(D)_{0}$, the following equation is obtained from (1).

$$
K=\frac{\left\{(P)_{0}+(R-1)(D)_{0}\right\} R}{1-R}
$$

Therefore,

$$
(D)_{0}=\frac{(P)_{0}}{1-R}-\frac{K}{R}
$$

By differenciating the equation (4) in respect to $(D)_{0}$,

$$
\left.\frac{d R}{d(D)_{0}}=1 /\left\{\frac{(P)_{0}}{(R-1)^{2}}+\frac{K}{R^{2}}\right\}\right\rangle 0
$$

is given. This shows that the ratio of $(D)$ to $(D)_{0}$ increases with the increase of $(D)_{0}$. Thus, the authors have come to the conclusion that the increase of the relative quantum yield is based on the increase of the portion of free dye which is capable of photosensitization. In the higher range of the concentration of dye, concentration quenching will predominate in the photosensitized oxidation with the decrease of the rate of the reaction or quantum yield.

Effect of pH on the Decomposition of Tryptophan Residue of Ovalbumin.

The increase of $\mathrm{pH}$ accelerates the decomposition of tryptophan residue of ovalbumin (Fig. 7). The curve shown in this figure is very similar with the acid-base titration curve of ovalbumin of which $\mathrm{pK}$ is 4.7 . This shows that ovalbumin with negative charge is more susceptible to photosensitized oxidation by the methylene blue than one with positive charge. Over the range of $\mathrm{pH}$ from 2 to 10 , methylene blue remains as cation. Therefore, the 


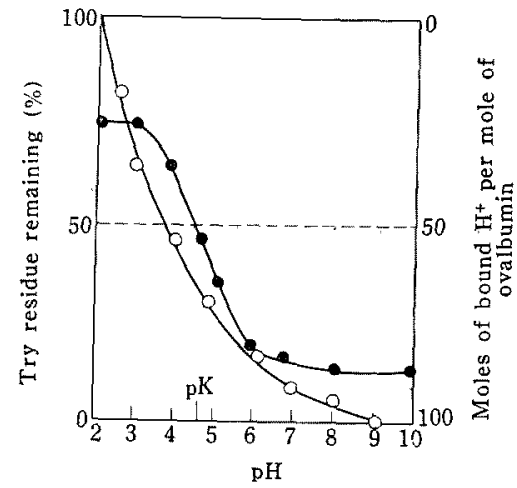

FIG. 7. Effect of $\mathrm{pH}$ on the Decomposition of Tryptophan Residue of Ovalbumin at $30^{\circ} \mathrm{C}$.

Reaction mixture:

Ovalbumin $\quad 10 \mathrm{mg} / \mathrm{ml}$

Methylene blue $\quad 10^{-4} \mathrm{M}$

Ionic strength $\quad 0.1$

- - Try residue remaining

- - Titration curve of ovalbumin.

acceleration of photosensitized oxidation by the increase of $\mathrm{pH}$ may be ascribed to the increase of the interaction between dye molecules and protein. If so, this is contrary to the assumption mentioned above stating that dye molecules adsorbed on the surface of protein are inactive for photosensitization. The similar dependences on $\mathrm{pH}$, however, were observed in the methylene blue sensitization on many free amino acids by Weil et al. ${ }^{12 !}$ In addition, Slyuterman also observed that the rise of $\mathrm{pH}$ accelerated the decomposition of histidine using proflavin, which is electricaly neutral, and the reaction rate was dependent on the dissociation of imidazole ring. ${ }^{271}$ And he came to the conclusion that the photosensitized oxidation was electrophyllic reaction. This also agrees with the theory presented by Kopecky et al. who dealt with the photosensitized oxidation of olefin compounds using methylene blue, eosine, riboflavin and chlorophyll as sensitizers. ${ }^{28 /}$ By taking

27) L. A. AE. Sluyterman, Biochim. Biophys. Acta, 60, 557 (1962)

28) K. R. Kopecky and H. H. Reich, Can. J. Chem., 43, 2265 (1965). these facts into consideration, it is reasonable that the acceleration of the decomposition of tryptophan residue of ovalbumin is not always based on the increase of the adsorption of dye molecules on protein.

Prevention of the Decomposition of Tryptophan Residue of Ovalbumin.

1) Effect of ascorbic acid. Ascorbic acid reduces the rate of the photodecomposition of tryptophan residue and breaks down itself. Such may be also the case with the protective effect by tyrosine. Dehydroascorbic acid does not show such effect at all. Ascorbic acid itself is decomposed effectively by the photosensitized action of dye resulting in dehydroascorbic acid. Fig. 8 shows the inhibiting

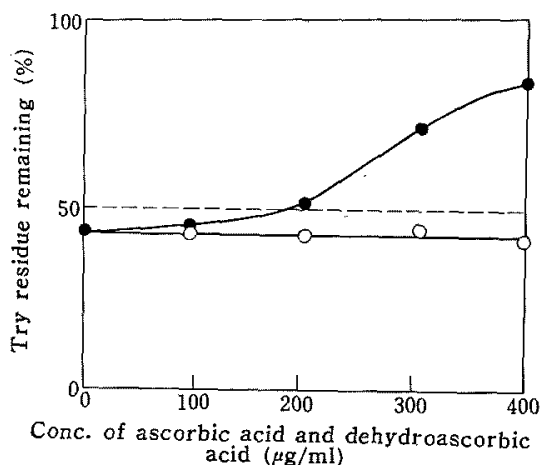

FIG. 8. Effect of Ascorbic Acid on the Decomposition of Tryptophan Residue of Ovalbumin at $30^{\circ} \mathrm{C}$.

Reaction mixture:

$$
\text { Ovalbumin } \quad 10 \mathrm{mg} / \mathrm{ml}
$$

Methylene blue $\quad 10^{-4} \mathrm{M}$

Acetate buffer $\quad 0.1 \mathrm{M}(\mathrm{pH} 6.0)$

Illuminated for three hrs.

- Ascorbic acid

-O- Dehydroascorbic acid.

effect on the decomposition of tryptophan residue of ovalbumin. The extent of inhibition increases with the increase of the amount of ascorbic acid added.

2) Illumination under anaerobic condition. Absence of oxygen results in no change of tryptophan content in ovalbumin, but methylene blue suffered from photobleaching (Fig. 9). Photobleached dye restored orignal color when 


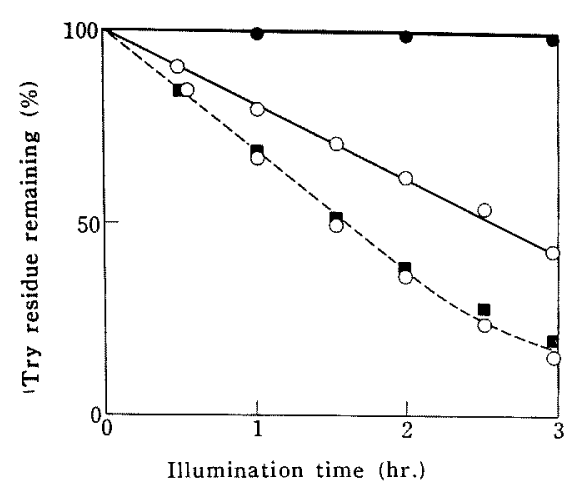

FIG. 9. Dependence on Gas Phase.

Reaction mixture:

$\begin{array}{ll}\text { Ovalbumin } & 10 \mathrm{mg} / \mathrm{ml} \\ \text { Methylene blue } & 10^{-4} \mathrm{M} \\ \text { Acetate buffer } & 0.1 \mathrm{M}(\mathrm{pH} 6.0)\end{array}$

$-\mathrm{N}_{2}$

- - Stood open to air

--- Aeration

-.-.- Bubbled with $\mathrm{O}_{2}$.

opened to the air. As known from other reports, amino acids and proteins are photodecomposed in the presence of oxygen alone.

3) Effect of anionic detergent. Addition of sodium lauryl sulfate (SLS) has strong effect on reduction of the decomposition of tryptophan residue of ovalbumin (Fig. 10). SLS as well as urea are well known as a good denaturant for protein. Both make protein structure random, but the effect of SLS is contrary to that of urea. SLS makes an ionic bond with a cationic group in protein even

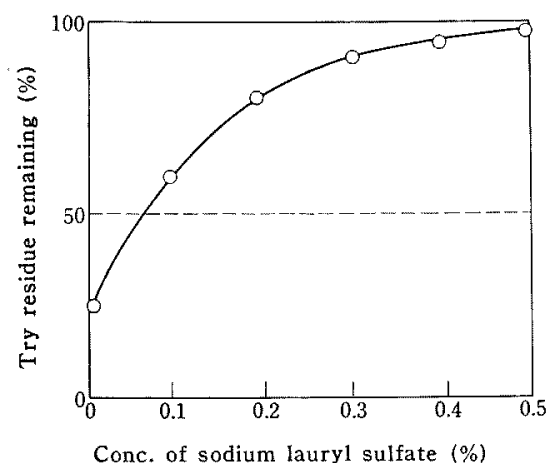

FIG. 10. Effect of Detergent on the Decomposition of Tryptophan Residue of Ovalbumin.

The reaction mixture was the same as shown in Fig. 9.

Illuminated for three hrs.

when the total charge is negative. ${ }^{29,301}$ The results given in this figure suggest that the bonding of SLS with a long alkyl chain to a cationic group of ovalbumin may mask photosusceptible indole group from photosensitized oxidation. This effect is very interesting, but further investigation will be necessary.

Acknowledgements. The authors wish to express their thanks to Prof. M. Fujimaki, the University of Tokyo, for his kind guidance and suggestions.

29) I. M. Klotz, F. W. Walker and R. B. Pivan, J. Am. Chem. Soc., 68, 1486 (1946).

30) F. Karush and M. Sonenberg, ibid., 71, 1369 (1949). 